\title{
ON LOWER BOUNDS FOR THE RADICAL OF A BLOCK IDEAL IN A FINITE $p$-SOLVABLE GROUP
}

\author{
by SHIGEO KOSHITANI
}

(Received 28th April 1983)

Dedicated to Professor Hirosi Nagao on his 60th birthday

Let $F$ be any field of characteristic $p>0, G$ a finite $p$-solvable group, $p^{a}$ the order of Sylow $p$-subgroups of $G, F G$ the group algebra of $G$ over $F$, and $J(F G)$ the Jacobson radical of $F G$. Following Wallace [11] we write $t(G)$ for the least integer $t \geqq 1$ such that $J(F G)^{t}=0$.

D. A. R. Wallace $[11]$ proved that

$$
t(G) \geqq a(p-1)+1
$$

The purpose of the present paper is to generalize the above result as follows: Let $B$ be a block ideal of $F G$ with defect $d$, and let $t(B)$ be the least integer $t \geqq 1$ such that $J(B)^{t}=0$ where $J(B)$ is the Jacobson radical of $B$. Then

$$
t(B) \geqq d(p-1)+1
$$

Since the defect groups of the principal block ideal of $F G$ are Sylow $p$-subgroups of $G$, our result is a generalization of that of Wallace.

We use the following notation and terminology. Throughout this paper we fix a field $F$ of characteristic $p>0$ and a finite group $G$, all modules are finitely generated right modules, and all groups are finite. For an Artinian ring $R$ and an integer $n \geqq 1$ let us denote by $\operatorname{Mat}(n, R)$ the full matrix ring of degree $n$ over $R$, by $Z(R)$ the centre of $R$, by $J(R)$ the Jacobson radical of $R$, and by $t(R)$ the least integer $t \geqq 1$ such that $J(R)^{t}=0$. In particular, we write $t(G)$ for $t(F G)$. Following [8, §2] we call $B \leftrightarrow e$ a block of $F G$ if $e$ is a centrally primitive idempotent of $F G$ such that $B=F G e$, and in this case we call $B$ a block ideal of $F G$. When $B$ is a block ideal of $F G$, we write $\delta(B)$ for a defect group of $B$ and $d(B)$ for the defect of $B$, i.e. $|\delta(B)|=p^{d(B)}$ (cf. [9, p. 211] and [8, Definition 3.9]), and we say that $B$ has full defect if $\delta(B)$ is a Sylow $p$-subgroup of $G$. When $H \triangleleft G$ and $b \leftrightarrow f$ is a block of $F H$, we write $T_{G}(b)$ or $T_{G}(f)$ for the inertia group of $b \leftrightarrow f$ in $G$, that is to say, $T_{G}(b)=T_{G}(f)=\left\{x \in G \mid x^{-1} f x=f\right\}$. If $H \triangleleft G$ and if $B$ and $b$ are block ideals of $F G$ and $F H$, respectively, then we say that $B$ covers $b$ in the sense of $[8, \S 6]$ (cf. [2, p. 196]). When $M_{R}$ is an $R$-module, we write $\operatorname{End}\left(M_{R}\right)$ for the ring of all $R$-moduleendomorphisms of $M_{R}$. We write $Z(G)$ for the centre of $G$. We use the notation $O_{p}(G)$, $O_{p}(G)$ and $O_{p^{\prime}, p}(G)$ as in [1, p. 397]. Further notation and terminology follow the books of Dornhoff [1] and Gorenstein [5]. 
First of all, we state Fong's results ([3], [4]) which are useful in the proof of our main result.

Lemma 1 (Fong). Assume that $F$ is an algebraically closed field of characteristic $p>0$. Let $H \triangleleft G$, let $b \leftrightarrow f$ be a block of $F H$, and let $T=T_{G}(f)$. Let $G=\bigcup_{i=1}^{t} \operatorname{Tg}_{i}$ be a coset decomposition of $T$ in $G$, let $f_{i}=g_{i}^{-1} f g_{i}$ for each $i$, and let $e=\sum_{i=1}^{t} f_{i}$. Then we have the following:

(1) $f$ is a central idempotent of FT.

(2) $f_{1}, \ldots, f_{t}$ are pairwise orthogonal centrally primitive idempotents of $F H$.

(3) $e$ is a central idempotent of $F G$ and $e f=f e=f$.

(4) $F G f$ is a free right $F T f$-module of rank $t$.

(5) $\operatorname{End}\left(F G f_{F T f}\right) \cong \operatorname{Mat}(t, F T f)$ as $F$-algebras.

(6) For each $x \in F G e$ and $y \in F G f$, define $\varphi(x) \in \operatorname{End}\left(F G f_{F T f}\right)$ by $[\varphi(x)](y)=x y$. Then $\varphi: F G e \rightarrow \operatorname{End}\left(F G f_{F T f}\right)$ is an $F$-algebra-isomorphism.

(7) Let $\widetilde{B}_{1} \leftrightarrow \tilde{e}_{1}, \ldots, \widetilde{B}_{m} \leftrightarrow \tilde{e}_{m}$ be blocks of FT such that $f=\sum_{j=1}^{m} \tilde{e}_{j}$, and let $B_{1} \leftrightarrow e_{1}, \ldots, B_{n} \leftrightarrow e_{n}$ be blocks of FG such that $e=\sum_{k=1}^{n} e_{k}$. Then

(i) $m=n, \widetilde{B}_{1}, \ldots, \widetilde{B}_{m}$ are all block ideals of $F T$ which cover $b$, and $B_{1}, \ldots, B_{m}$ are all block ideals of $F G$ which cover $b$.

For suitable indexing of $\widetilde{B}_{j}$ and $B_{j}$, we get for each $j=1, \ldots, m$ that

(ii) $B_{j} \cong \operatorname{Mat}\left(t, \widetilde{B}_{j}\right)$ as $F$-algebras.

(iii) $\tilde{e}_{j} e_{j}=e_{j} \tilde{e}_{j}=\tilde{e}_{j}$.

(iv) $\widetilde{B}_{j}^{G}=B_{j}$.

(v) $\delta\left(B_{j}\right) \cong \delta\left(\widetilde{B}_{j}\right)$.

(vi) If $\tilde{S}$ is a simple FT-module in $\tilde{B}_{j}$, then $\tilde{S}^{G}$ is a simple FG-module in $B_{j}$ where $\tilde{S}^{G}=\tilde{S} \otimes_{F T} F G$.

Proof. (1) Obvious.

(2) Clearly, $f_{1}, \ldots, f_{t}$ are distinct centrally primitive idempotents of $F H$. Hence $f_{i} f_{j}=0$ if $i \neq j$.

(3) By (2), $e^{2}=e$ and $e f=f e=f$. Hence $e \neq 0$. Take any $g \in G$. Since $G=\bigcup_{i=1}^{t} T g_{i} g$ is also a coset decomposition of $T$ in $G$, we get $g^{-1} e g=e$, so that $e \in Z(F G)$.

(4) Since $F G f=\bigoplus_{i=1}^{t} g_{i}^{-1} F T f$ and $g_{i}^{-1} F T f \cong F T f$ as right $F T f$-modules for all $i$, we get (4).

(5) Trivial from (4).

(6) Obviously, $\varphi$ is well-defined. Let $E=\operatorname{End}\left(F G f_{F T f}\right)$. By (3), $\varphi(e)$ is the identity map of $F G f$, so that $\varphi$ is an $F$-algebra-homomorphism.

Assume $\varphi(x)=0$ for some $x \in F G e$. Then $x y=0$ for all $y \in F G f$. Hence $0=$ $\sum_{i=1}^{t} x g_{i}^{-1} f g_{i}=x e=x$. Thus $\varphi$ is monomorphic.

Take any $\sigma \in E$. Let $x=\left[\sum_{i=1}^{t} \sigma\left(g_{i}^{-1} f\right) g_{i} f_{i}\right] e \in F G e$. Then by (2), $x=\sum_{i=1}^{t} \sigma\left(g_{i}^{-1} f\right) g_{i} f_{i}$. 
Let $y \in F G f$. Then we can write $y=\sum_{j=1}^{t} g_{i}^{-1} s_{j}$ where $s_{j} \in F T f$. By (1), $f s_{j}=s_{j} f=s_{j}$. Thus

$$
\sigma(y)=\sum_{j} \sigma\left(g_{j}^{-1} s_{j}\right)=\sum_{j} \sigma\left(g_{j}^{-1} f s_{j}\right)=\sum_{j} \sigma\left(g_{j}^{-1} f\right) s_{j}
$$

since $\sigma \in E$. On the other hand, since $f_{s_{j}}=s_{j}$, we get by (2)

$$
\begin{aligned}
{[\varphi(x)](y) } & =x y=\sum_{i} \sum_{j} \sigma\left(g_{i}^{-1} f\right) g_{i} f_{i} g_{j}^{-1} s_{j} \\
& =\sum_{i} \sum_{j} \sigma\left(g_{i}^{-1} f\right) g_{i} f_{i}\left(g_{j}^{-1} f g_{j}\right) g_{j}^{-1} s_{j} \\
& =\sum_{i} \sigma\left(g_{i}^{-1} f\right) g_{i} f_{i} g_{i}^{-1} s_{i}=\sum_{i} \sigma\left(g_{i}^{-1} f\right) f s_{i} \\
& =\sum_{i} \sigma\left(g_{i}^{-1} f\right) s_{i} .
\end{aligned}
$$

Hence $\sigma(y)=[\varphi(x)](y)$, so that $\sigma=\varphi(x)$. Hence $\varphi$ is epimorphic.

(7) By [2, V Lemma 3.3] (cf. $[8, \S 6]$ ), $B_{1}, \ldots, B_{n}$ are all block ideals of $F G$ which cover $b$. Similarly, $\widetilde{B}_{1}, \ldots, \widetilde{B}_{m}$ are all block ideals of $F T$ which cover $b$. Then $m=n$ by [2, V Theorem 2.5]. Since $F G e=\bigoplus_{j=1}^{m} F G e_{j}$ and $F T f=\bigoplus_{j=1}^{m} F T \tilde{e}_{j}$, by (5) and (6) for suitable indexing of $e_{j}$ and $\tilde{e}_{j}$ we have the $F$-algebra-isomorphisms

$$
\begin{aligned}
& F G e_{j} \stackrel{\approx}{\longrightarrow} \operatorname{End}\left[\left(F G \tilde{e}_{j}\right)_{F T \tilde{e}_{j}}\right] \stackrel{\approx}{\longrightarrow} \operatorname{Mat}\left(t, F T \tilde{e}_{j}\right) \\
& \omega \\
& x \longmapsto[\varphi(x): y \mapsto x y]
\end{aligned}
$$

for $j=1, \ldots, m$. Let us fix any $j$. Since $e_{j}$ is the unit element of the ring $F G e_{j}, \varphi\left(e_{j}\right)$ is the identity map of $F G \tilde{e}_{j}$. Hence $\tilde{e}_{j} e_{j}=e_{j} \tilde{e}_{j}=\tilde{e}_{j}$. Let $\tilde{S}$ be a minimal right ideal of $\widetilde{B}_{j}=F T \tilde{e}_{j}$. Then

$$
\tilde{S}^{G} e_{j}=\tilde{S} F G e_{j}=\tilde{S} \tilde{e}_{j} F G e_{j}=\tilde{S} \tilde{e}_{j} e_{j} F G=\tilde{S} \tilde{e}_{j} F G=\tilde{S} F G=\tilde{S}^{G}
$$

Hence $\tilde{S}^{G} \subseteq F G e_{j}=B_{j}$. Thus the correspondence $\widetilde{B}_{j} \leftrightarrow B_{j}$ is the same as that of $[2, \mathrm{~V}$ Theorem 2.5]. Therefore (7) is proved by [2, V Theorem 2.5].

Lemma 2 (Fong). Assume that $F$ is an algebraically closed field of characteristic $p>0$. Let $H \triangleleft G$ such that $p \nmid|H|$, and let $b$ be a block ideal of $F H$ covered by a block ideal $B$ of $F G$. If $T_{G}(b)=G$, then there are a finite group $\tilde{G}$ and an exact sequence

$$
1 \longrightarrow Z \longrightarrow \tilde{G} \stackrel{f}{\longrightarrow} G \longrightarrow 1
$$

which satisfy the following:

(1) $Z$ is cyclic, $Z \subseteq Z(\tilde{G})$ and $\left.|Z||| H\right|^{2}$.

(2) $\tilde{G}$ has a normal subgroup $\tilde{H}$ such that $\tilde{H} \cong H$ and $Z \tilde{H}=Z \times \tilde{H}=f^{-1}(H)$. 
(3) $F(\tilde{G} / \tilde{H})$ has a block ideal $B^{*}$ such that $B \cong \operatorname{Mat}\left(n, B^{*}\right)$ as $F$-algebras for an integer $n \geqq 1$ and that $\delta\left(B^{*}\right) \cong \delta(B)$.

(4) Let $X=\tilde{G} / \tilde{H}$. Especially, if $G$ is p-solvable, $p|| G \mid$ and $H=O_{p^{\prime}}(G)$, then we get the following:

(i) $X$ is also p-solvable.

(ii) $O_{p},(X) \subseteq Z(X)$.

(iii) $X$ has a normal p-subgroup $Q$ such that $O_{p^{\prime}, p}(X)=O_{p^{\prime}}(X) \times Q$.

(iv) $O_{p}(X) \neq 1$.

(v) Every block ideal of $F X$ has full defect.

Proof. By [2, X Lemma 1.1 and Theorem 1.2], [12, §1] and [10, Theorem 2], we have an exact sequence $\left({ }^{*}\right)$ which satisfies (1), (2) and (3).

(4) (i) is clear. Since $p \nmid|\tilde{H}|, O_{p^{\prime}}(X)=O_{p^{\prime}}(\tilde{G}) / \tilde{H}$. By (1) and (2), $O_{p^{\prime}}(\tilde{G})=Z \times \tilde{H}$. Hence $O_{p},(X) \subseteq Z(X)$ by (1). Since $O_{p^{\prime}, p}(X)$ is $p$-nilpotent, we get (iii) from (ii). Since $p|| X \mid$, by (i) and (iii) we have $1 \neq Q \subseteq O_{p}(X)$ (cf. [2, p. 416]). (v) is obtained from [2, X Lemma 1.4].

The next lemma has been essentially proved by Wallace [11, Theorem 2.4].

Lemma 3 (Wallace). Let $F$ be any field of characteristic $p>0$ and $P$ a normal p-subgroup of $G$, and let $\bar{G}=G / P$. Let $F G \stackrel{f}{\rightarrow} F \bar{G}$ be the canonical ring-epimorphism such that $f(g)=g P$ for each $g \in G$, and let $B \leftrightarrow e$ be a block of $F G$. Then we can write $f(B)=\bigoplus_{i=1}^{n} \bar{B}_{i}$ for an integer $n \geqq 1$ where each $\bar{B}_{i}$ is a block ideal of $F \bar{G}$. Moreover, we have the following:

(1) $t(B) \leqq t(P) \cdot m$ where $m=\max \left\{t\left(\bar{B}_{i}\right) \mid i=1, \ldots, n\right\}$.

(2) $t(B) \geqq t(P)+t\left(\bar{B}_{i}\right)-1$ for all $i=1, \ldots, n$.

Proof. The proof is similar to that of Wallace [11, Theorem 2.4]. Let $G=\bigcup_{j=1}^{q} g_{j} P$ be a coset decomposition of $P$ in $G$. Then $F G=\bigoplus_{j=1}^{q} g_{j} F P$, so that $F G \cdot J(F P)=$ $\bigoplus_{j=1}^{q} g_{j} J(F P)$. By [8, Lemma 4.5] and [6, Theorem 1.2], Ker $f=J(F P) F G=$ $F G \cdot J(F P)$, so that $\operatorname{Ker} f$ is a nilpotent ideal of $F G$. Hence $\operatorname{Ker} f \subseteq J(F G)$. Then $f(e) \neq 0$ since $\operatorname{Ker} f$ is nilpotent. Thus we can write $f(e)=\sum_{i=1}^{n} \bar{e}_{i}$ for an integer $n \geqq 1$ where each $\bar{e}_{i}$ is a centrally primitive idempotent of $F \bar{G}$. Let $\bar{B}_{i}=F \bar{G} \bar{e}_{i}$ for each $i$, then $f(B)$ $=\bigoplus_{i=1}^{n} \bar{B}_{i}$.

(1) Let $\tilde{f}=\left.f\right|_{B}: B \rightarrow f(B)$. Then $\operatorname{Ker} \bar{f}=\operatorname{Ker} f \cap B=(\operatorname{Ker} f) e=J(F P) B$, so that $\operatorname{Ker} \tilde{f}=$ $J(F P) B=B \cdot J(F P) \subseteq J(B)$. Thus $f$ induces a ring-isomorphism

$$
\bigoplus_{i=1}^{n} \bar{B}_{i}=f(B) \cong B / \operatorname{Ker} \tilde{f}=B / J(F P) B
$$

Since $J[B / J(F P) B]=[J(B)+J(F P) B] / J(F P) B=J(B) / J(F P) B$, we have

$$
\bigoplus_{i=1}^{n} J\left(\bar{B}_{i}\right)=J\left(\bigoplus_{i=1}^{n} \bar{B}_{i}\right) \cong J(B) / J(F P) B
$$


Then since $\left[\bigoplus_{i=1}^{n} J\left(\bar{B}_{i}\right)\right]^{m}=\bigoplus_{i} J\left(\bar{B}_{i}\right)^{m}=0$, we get $J(B)^{m} \subseteq J(F P) B=B \cdot J(F P)$. Thus we have $J(B)^{m \cdot t(P)}=0$, so that $t(B) \leqq m \cdot t(P)$.

(2) Fix any $i(1 \leqq i \leqq n)$, and let $\bar{B}=\bar{B}_{i}$ and $t=t(\bar{B})$. Since $J(\bar{B})^{t-1} \neq 0$, we get

$$
f\left[J(B)^{t-1}\right]=[\tilde{f}(J(B))]^{t-1}=[J(\tilde{f}(B))]^{t-1}=\bigoplus_{k=1}^{n} J\left(\bar{B}_{k}\right)^{t-1} \neq 0 .
$$

Then $J(B)^{t-1} \notin \operatorname{Ker} f=J(F P) B$, so that there is some $w \in J(B)^{t-1}-J(F P) B$. We can write $w=\sum_{j=1}^{q} g_{j} s_{j}$ where $s_{j} \in F P$. Clearly, $w \notin J(F P) F G=F G \cdot J(F P)$. Thus we may assume $s_{1} \notin J(F P)$. We can write $s_{1}=\sum_{x \in P} c_{x} x$ where $c_{x} \in F$. Without the assumption that $F$ is algebraically closed, the result of Wallace [11, Lemma 2.3] holds (cf. [6]). Hence by [11, Lemma 2.3(3)], $\sum_{x \in P} c_{x} \neq 0$. Let $\hat{P}=\sum_{x \in P} x$ in $F G$.

Next, we want to claim that $w \hat{P} \neq 0$. Suppose $w \hat{P}=0$. Since $w \hat{P}=\left(\sum_{j} g_{j} s_{j}\right) \hat{P}=\sum_{j} g_{j}\left(s_{j} \hat{P}\right)$ and since $s_{j} \hat{P} \in F P$ for all $j$, we have $s_{j} \hat{P}=0$ for all $j$. Thus $0=s_{1} \hat{P}=\left(\sum_{x \in P} c_{x} x\right) \hat{P}=$ $\left(\sum_{x \in P} c_{x}\right) \hat{P}$, so that $\sum_{x \in P} c_{x}=0$, a contradiction.

Hence $w \hat{P} \neq 0$. Since $J(F P)^{t(P)-1}=F \hat{P}$ by $[11$, Lemma $2.3(2)]$ and since $e \cdot J(F P)^{h}=$ $J(F P)^{h} e \subseteq J(B)^{h}$ for any integer $h \geqq 0$, we have $w \hat{P} \in J(B)^{t+t(P)-2}$. Thus $t(B) \geqq t+t(P)-1$.

Now, we are ready to prove the following main result of this paper.

Theorem. Let $F$ be any field of characteristic $p>0, G$ a finite p-solvable group and $B$ a block ideal of FG with defect $d$. Then we have

$$
t(B) \geqq d(p-1)+1
$$

Proof. Let $E$ be the algebraic closure of $F$. By [8, Lemma 12.9], we can write $E \otimes_{F} B=$ $\bigoplus_{i=1}^{n} B_{i}^{*}$ for an integer $n \geqq 1$ where each $B_{i}^{*}$ is a block ideal of $E G$ with the same defect $d$. By [8, Corollary 12.12], for any integer $m \geqq 1 E \otimes_{F} J(B)^{m}=J\left(E \otimes_{F} B\right)^{m}=\bigoplus_{i} J\left(B_{i}^{*}\right)^{m}$. So $t(B) \geqq t\left(B_{i}^{*}\right)$ for all $i$. Thus we may assume that $F$ is algebraically closed.

We prove the theorem by double induction on $d$ and $|G|$.

If $d=0$, then $J(B)=0$ (cf. [1, Theorem 62.5]), so that it is easy. Thus we may assume $d \geqq 1$, so that $p|| G \mid$.

If $G=\delta(B)$, then $B=F G$, so that it is proved by [11, Lemma 2.3(1)].

Let $H=O_{p},(G)$. Then there is a block ideal $b$ of $F H$ covered by $B$. Let $T=T_{G}(b)$. By Lemma $1(7), F T$ has a block ideal $\tilde{B}$ with the same defect $d$ and $t(\tilde{B})=t(B)$.

If $G \neq T$, then since $|T|<|G|$ we get the result by induction. Hence we may assume $G=T$.

Then by Lemma 2 , there is a finite $p$-solvable group $X$ such that $O_{p}(X) \neq 1$ and $F X$ has a block ideal $B^{*}$ with the same defect $d$ and $t\left(B^{*}\right)=t(B)$. Let $P=O_{p}(X),|P|=p^{r}$ and $\bar{X}=X / P$. By $[2, \mathrm{~V}$ Lemma 4.4] and Lemma $3(2), F \bar{X}$ has a block ideal $\bar{B}$ with defect $d-r$ and $t\left(B^{*}\right) \geqq t(P)+t(\bar{B})-1$. By $[11$, Lemma $2.3(1)], t(P) \geqq r(p-1)+1$. Since $d-r<d$, we get by induction that $t(\bar{B}) \geqq(d-r)(p-1)+1$. Therefore

$$
t(B)=t\left(B^{*}\right) \geqq t(P)+t(\bar{B})-1 \geqq d(p-1)+1 .
$$

This completes the proof of the theorem. 
Corollary (Wallace [11, Theorem 3.3]). Let $F$ be any field of characteristic $p>0, G a$ finite $p$-solvable group, $p^{a}$ the order of $S y l o w$-subgroups of $G$, and $B_{0}(G)$ the principal block ideal of $F G$. Then

$$
t(G) \geqq t\left(B_{0}(G)\right) \geqq a(p-1)+1
$$

Proof. Since $d\left(B_{0}(G)\right)=a$, it is clear from Theorem.

Remark. W. Willems [13] has also improved the result of Wallace [11, Theorem 3.3] (cf. [13, 3.5 Theorem and 3.6 Corollary]). But our theorem is not contained in that of Willems.

Let $G$ be a finite $p$-solvable group such that $G$ has no proper normal subgroups of index prime to $p$ and that $F G$ has a non-principal block ideal $B$ with full defect $d$, so that $v_{p}(|G|)=d$ where we use the notation $v_{p}(n)$ for an integer $n \geqq 1$ as in $[1, \mathrm{p} .376]$. Let $S$ be a simple $F G$-module in $B$, and let $K=\operatorname{Ker} S$ where $\operatorname{Ker} S$ is the kernel of $S$ in $G$.

Assume $v_{p}(|K|)=d$. Then there is a Sylow $p$-subgroup $D$ of $G$ such that $D \subseteq K$. Let $M=\left\langle g^{-1} D g \mid g \in G\right\rangle$. Since $K \triangleleft G, M \leqq K$. Since $M \triangleleft G$ and $p \nmid|G: M|, G=M$. Thus $K=G$, so that $S$ is the trivial $F G$-module. Hence $B$ is the principal block ideal of $F G$, a contradiction.

Thus for any simple $F G$-module $S$ in $B$ we get $v_{p}(|\operatorname{Ker} S|)<d$, so that $v_{p}(|\operatorname{Ker} S|) \cdot(p-1)$ $+1<d(p-1)+1$. Thus our theorem is not contained in [13, 3.5 Theorem (b)].

In fact, there is a finite $p$-solvable group $G$ which satisfies the above conditions. See our previous example [7, Example 3 (pp. 229-230)].

\section{REFERENCES}

1. L. Dornhoff, Group Representation Theory (part B, Marcel Dekker, New York, 1972).

2. W. FErT, The Representation Theory of Finite Groups (North-Holland, New York, 1982).

3. P. Fong, On the characters of p-solvable groups, Trans. Amer. Math. Soc. 98 (1961), 263284.

4. P. Fong, Solvable groups and modular representation theory, Trans. Amer. Math. Soc. 103 (1962), 484-494.

5. D. Gorenstein, Finite Groups (Harper \& Row, New York, 1968).

6. S. A. Jennings, The structure of the group ring of a p-group over a modular field, Trans. Amer. Math. Soc. 50 (1941), 175-185.

7. S. Koshitan, Group algebras of finite $p$-solvable groups with radicals of the fourth power zero, Proc. Royal Soc. Edinburgh 92A (1982), 205-231.

8. G. O. MichleR, Blocks and centers of group algebras. Lectures on Rings and Modules (Lecture notes in math. 246, Springer, Berlin, 1972), 429-563.

9. A. Rosenberg, Blocks and centres of group algebras, Math. Z. 76 (1961), 209-216.

10. Y. Tsushima, On the second reduction theorem of P. Fong, Kumamoto J. Science (Math.) 13 (1978), 6-14.

11. D. A. R. Wallace, Lower bounds for the radical of the group algebra of a finite $p$-soluble group, Proc. Edinburgh Math. Soc. 16 (1968), 127-134. 
12. A. Watanabe, On Fong's reductions, Kumamoto J. Science (Math.) 13 (1979), 48-54.

13. W. Willems, On the projectives of a group algebra, Math. Z. 171 (1980), 163-174.

Department of Mathematics

Faculty OF SCIENCE

ChIBA UNIVERSITY

Yayol-Cho, ChiBa-CITY, 260

JAPAN 